\title{
Cactus protein degradation mediates Drosophila dorsal-ventral signaling
}

\author{
Marcia P. Belvin, Yishi Jin, ${ }^{1}$ and Kathryn V. Anderson ${ }^{2}$ \\ Division of Genetics, Department of Molecular and Cell Biology, University of California at Berkeley, Berkeley, California \\ 94720 USA
}

\begin{abstract}
Dorsal-ventral patterning in the Drosophila embryo relies on a signal transduction pathway that is similar to a signaling pathway leading to the activation of the mammalian transcription factor NF- $\mathrm{B}$. Stimulation of this Drosophila pathway on the ventral side of the embryo causes the nuclear translocation of Dorsal, the Drosophila NF-кB homolog. Cactus, like its mammalian homolog IкB, inhibits nuclear translocation by binding Dorsal and retaining it in the cytoplasm. We show that Cactus, like IKB, is rapidly degraded in response to signaling. More importantly, signal-dependent degradation of Cactus does not require the presence of Dorsal, indicating that Cactus degradation is a direct response to signaling, and that disruption of the Dorsal/Cactus complex is a secondary result of Cactus degradation. Mutant alleles of cactus that encode more stable forms of the protein block signaling, showing that efficient degradation is necessary for signaling. We find that Cactus protein stability is regulated by two independent processes that rely on different regions within the protein: signal-dependent degradation requires sequences in the amino terminus or ankyrin repeats, whereas signal-independent degradation of free Cactus requires the carboxy-terminal region of the protein that includes a PEST sequence.
\end{abstract}

[Key Words: Cactus; IкB; NF-кB; protein degradation; dorsal-ventral patterning]

Received January 17, 1995; revised version accepted February 27, 1995.

Signal transduction pathways that mediate the responses of eukaryotic cells to extracellular signaling molecules generally involve covalent modifications of cytoplasmic proteins. Protein phosphorylation, mediated either by receptor tyrosine kinases or by cytoplasmic kinases that associate with activated receptors, is the best understood of these signal-induced modifications. Recent data have suggested that a different kind of covalent change, protein degradation, may play an important role in at least one kind of signal transduction pathway, the pathway that leads to the activation of the transcription factor NF- $\mathrm{B}$.

NF- $\kappa$ B, a mammalian transcription factor originally identified in B cells, but subsequently found in several nonimmune cell types, is activated rapidly by a posttranslational mechanism in response to a variety of extracellular stimuli (Blank et al. 1992; Grilli et al. 1993). Inactive NF- $\mathrm{B}$ is retained in the cytoplasm by its inhibitor, IкB, and migrates into the nucleus when the NF$\kappa \mathrm{B} / \mathrm{I} \kappa \mathrm{B}$ complex dissociates in response to several cytokines, including interleukin-1 (IL-1) and tumor necrosis factor (TNF $\alpha$ ) (Sen and Baltimore 1986; Bauerle and Balimore 1988; Beg et al. 1993). IкB family members share a block of ankyrin repeats that mediate binding to NF- $\mathrm{B}$

${ }^{1}$ Present address: Department of Biology, Massachusetts Institute of Technology, Cambridge, Massachusetts 02139 USA.

${ }^{2}$ Corresponding author. and mask the NF- $\kappa$ B nuclear localization signal /Ganchi et al. 1992; Henkel et al. 1992; Beg and Baldwin 1993), providing an explanation for why NF- $\mathrm{B} / \mathrm{I} \kappa \mathrm{B}$ complexes remain cytoplasmic while free NF- $\mathrm{B}$ is nuclear.

Recent work using mammalian tissue culture cells has shown that IKB is rapidly degraded in response to signaling, and that this degradation correlates with NF- $\mathrm{B}$ activation (Beg et al. 1993; Henkel et al. 1993; Miyamoto et al. 1994; Palombella et al. 1994; Traenckner et al. 1994). Several calpain and proteasome inhibitors stabilize IкB and block NF- $\kappa \mathrm{B}$ activation, indicating that $\mathrm{I} \kappa \mathrm{B}$ degradation is necessary for activation of NF- $\mathrm{B}$ (Miyamoto et al. 1994; Traenckner et al. 1994; Lin et al. 1995). In some of these experiments, the stabilized form of $I_{\kappa} B$ is a more highly phosphorylated form, suggesting that phosphorylation of I $\mathrm{B}$ in the complex in response to signaling precedes its degradation.

The Drosophila dorsal-ventral signal transduction pathway is comprised of 12 known maternal effect genes. The end result of the activity of this pathway is the nuclear localization of the transcription factor Dorsal on the ventral side of the embryo. Three components of this pathway, Toll, Cactus, and Dorsal, are homologous to members of the IL-1/NF-kB pathway. The cytoplasmic domain of Toll, a transmembrane receptor protein, is homologous to the cytoplasmic domain of the mammalian IL-1 receptor (Hashimoto et al. 1988; Schneider et al. 1991). Dorsal and Cactus are homolo- 


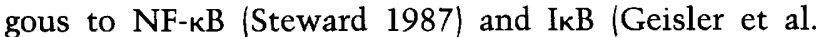
1992; Kidd 1992), respectively. Localized ventral activation of the Toll receptor causes disruption of the Dorsal/ Cactus complex and the subsequent nuclear localization of Dorsal, just as activation of the IL-1 receptor leads to the disruption of the NF- $\mathrm{BB} / \mathrm{I} \mathrm{KB}$ complex and the nuclear localization of NF-kB. Neither signal-induced phosphorylation nor signal-dependent degradation of the Drosophila IкB homolog Cactus has been observed previously (Whalen and Steward 1993). However, given the homology that exists between the Drosophila and mammalian pathways, it seems likely that degradation of Cactus could also be linked to the activation of Dorsal.

The early Drosophila embryo provides a unique opportunity to dissect the in vivo response of Cactus and Dorsal to signaling. By activating the pathway at a precise time by microinjection of the activating extracellular ligand, Spätzle, we show that Cactus, like IkB, is rapidly degraded in vivo in response to signaling. Taking advantage of Drosophila mutants, we show that signal-induced Cactus degradation can occur in the absence of Dorsal, indicating that degradation of the Cactus protein is a direct result of signaling, rather than a secondary consequence of separation of the Dorsal/Cactus complex. We find that mutant alleles of Cactus that increase its stability block signaling, confirming that destruction of Cactus is necessary for signaling. These mutant alleles also reveal that Cactus protein turnover is regulated by two independent processes: signal-independent degradation of free Cactus, which requires the carboxy-terminal PEST region of the protein, and signal-dependent degradation, which is mediated by more amino-terminal regions of the protein.

\section{Results}

Signaling causes rapid degradation of wild-type Cactus

To test whether Cactus, like $I_{\kappa} B$, is degraded in response to signaling, we activated the dorsal-ventral signaling pathway at a controlled time by injecting embryos with processed Spätzle, the putative Toll ligand (Morisato and Anderson 1994; Schneider et al. 1994; Fig. 1C). An activated, processed form of the Spätzle protein can activate Toll when it is injected into the extracellular (perivitelline) space of early embryos (Schneider et al. 1994). Therefore, to stimulate the pathway, we injected activated Spätzle protein into the perivitelline space of syncytial blastoderm embryos. In the 1- to 1.5 -hr embryos used, all of the components required for signaling are present, and localized endogenous signaling is just beginning. After a short incubation, the embryonic cytoplasm was harvested and the Cactus protein was analyzed by Western blot. We observed rapid degradation of Cactus in response to injection of activated Spätzle (Fig. 1A); $>75 \%$ of the Cactus protein was degraded in the first 15 min after injection. In control injections with water, Cactus was not detectably degraded in the $30 \mathrm{~min}$ following injection (data not shown).

To test whether the Cactus degradation that we observed was specific to the dorsal-ventral pathway, we repeated these injections into embryos from $\mathrm{Toll}^{-}\left(T 1^{-}\right)$ females. Because activated Spätzle is postulated to bind and activate the Toll receptor, it should not stimulate signaling in an embryo lacking Toll. Figure 1B shows that injection of activated Spätzle into $\mathrm{Tl}^{-}$embryos did not cause degradation of Cactus. Therefore, the signaldependent degradation of Cactus that we observe in
Figure 1. Degradation of Cactus in response to signaling. Response of $1.5-$ to 2-hr-old embryos from wild-type $(A)$ or $T^{-}, t u b^{-}$, and $\mathrm{pll}^{-}$females $(B) .(C)$ Schematic diagram of the order of action of genes in the pathway. For $A$ and $B$, embryos were injected in the perivitelline space with an activated Spätzle preparation. Embryonic cytoplasm was collected 5 or $15 \mathrm{~min}$ after injection and analyzed by Western blot, using an anti-Cactus antibody. The lanes labeled Uninj contain cytoplasm from uninjected embryos; the lanes marked $5^{\prime}$ and $15^{\prime}$ contain cytoplasm collected 5 and $15 \mathrm{~min}$ after injection of Spätzle protein. The arrows point to the Cactus protein. In wild-type embryos, Cactus protein disappeared rapidly after Spätzle injection $(A)$. In mutant embryos that lack Toll, Tube, or Pelle, there was no degradation of Cactus in response to Spätzle injection $(B)$. The high molecular weightband in each lane is a crossreacting band that can be used as an internal loading control. Control injections with water caused no degradation of Cactus (data not shown). The blot in $A$ resolves the two forms of Cactus: the predominant phosphorylated form (arrow), and the less abundant, more rapidly migrating unphosphorylated form. The upper band collapses to the lower band after treatment with calf intestinal phosphatase (Whalen and Steward 1993).

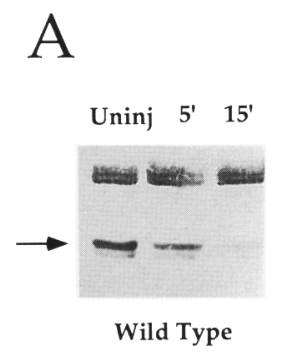

B
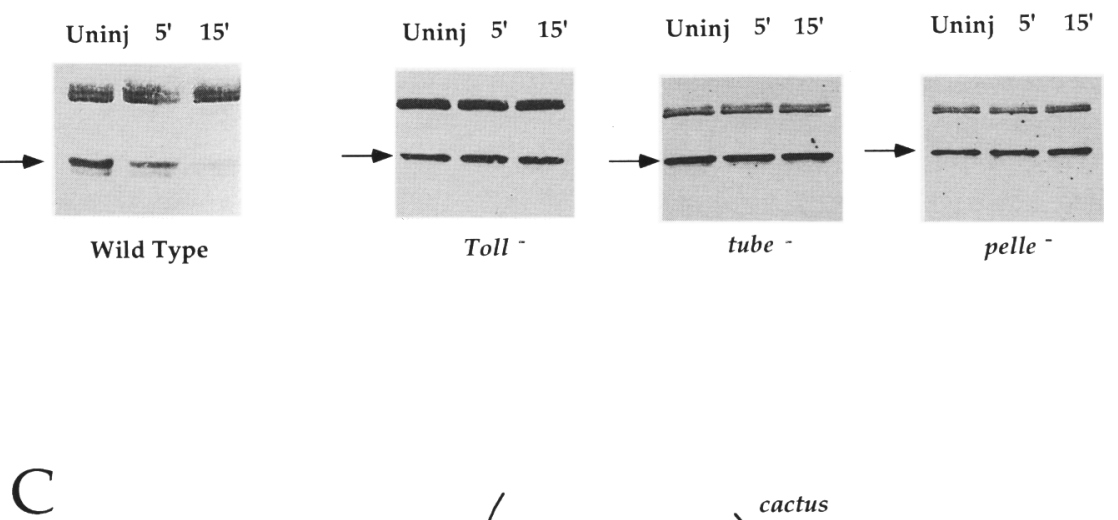
wild-type embryos is specific to the dorsal-ventral pathway. We also tested whether this degradation required the dorsal group genes tube and pelle, which act downstream of Toll and upstream of dorsal and cactus (Fig. 1C). The sequence of tube does not suggest a biochemical function (Letsou et al. 1991); pelle appears to encode a cytoplasmic serine/threonine kinase (Shelton and Wasserman 1993). We found that in embryos from tube $e^{-}$ $\left(t u b^{-}\right)$and pelle ${ }^{-}\left(p 11^{-}\right)$females, Cactus does not degrade in response to signaling (Fig. 1B). Therefore, signaldependent degradation of Cactus is mediated by all of the genetically defined components of the pathway that act between Spätzle and dorsal/cactus in the pathway.

\section{Cactus is rapidly degraded in response to signal even in the absence of Dorsal}

When the rapid degradation of $I_{\kappa} B$ in response to TNF $\alpha$ is blocked by calpain inhibitors, I $\mathrm{k} B$ becomes phosphorylated but does not dissociate from NF- $\mathrm{kB}$ (Miyamoto et al. 1994). One interpretation of this result is that the degradation of $I_{\kappa} B$ in response to signal takes place when it is in the complex. However, in the absence of inhibitors, it has not been shown whether degradation of IкB is a direct response to in vivo signaling or occurs as a secondary consequence of I $\mathrm{k}$ becoming separated from NF$\kappa \mathrm{B}$. To differentiate between these two possibilities for Cactus and Dorsal, we wanted to assay the response of Cactus to injection of activated Spätzle into embryos lacking Dorsal protein. If Cactus is a direct target for signaling, then activation of the signaling pathway should cause rapid degradation of Cactus even in the absence of Dorsal. If Cactus is degraded only as a consequence of being separated from Dorsal, then the level of Cactus protein in the absence of Dorsal should be unresponsive to signaling.

A complication of this experiment is that that there is no detectable maternal Cactus protein in embryos or ovaries lacking Dorsal (Fig. 2, lane 1; Whalen and Steward 1993; Kidd 1992), suggesting that Cactus is unstable when not bound to Dorsal even prior to signaling. We found, however, that it is possible to produce Cactus protein in embryos lacking Dorsal protein, that is, embryos laid by dorsal ${ }^{-}\left(\mathrm{dl}^{-}\right)$females, by injecting in vitrosynthesized cactus RNA into the cytoplasm of these embryos and allowing translation in vivo (Fig. 2, lanes 2,4). To test the effect of signaling, activated Spätzle was injected into the perivitelline space of the embryos after translation of Cactus had begun (Fig. 2, lanes 3,5). We observed that Cactus was degraded in response to signaling at roughly the same rate in the embryos from $\mathrm{dl}^{-}$ females as in embryos from wild-type females (cf. Fig. 2, lanes 2-5 with Fig. 1A), whereas it continued to accumulate in the absence of signaling (Fig. 2, lanes 2,4). We conclude that free Cactus is as effective a substrate for signal-dependent degradation as Cactus in the DorsalCactus complex, indicating that Cactus degradation is a direct consequence of signaling.

This experiment also revealed that two kinds of degradation control the level of Cactus protein in the em-

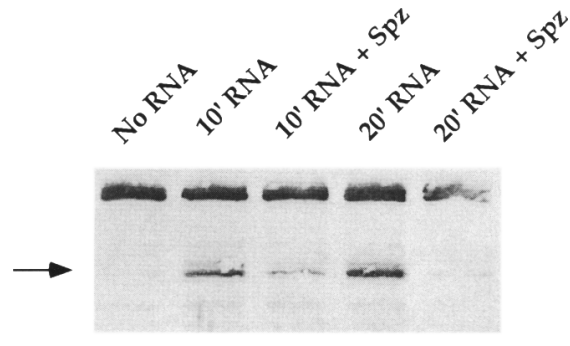

dorsal- embryos

Figure 2. Degradation of Cactus in response to signaling in the absence of Dorsal. To test the effect of signaling on Cactus protein in the absence of Dorsal, 1.5- to 2-hr-old embryos laid by $\mathrm{dl}^{-}$females were injected with cactus transcripts. (Lane 1) Uninjected $\mathrm{dl}^{-}$embryos contain no detectable Cactus protein. Following a 5-min incubation to allow translation of the injected RNA, activated Spätzle was injected into the perivitelline space of the experimental embryos (lanes 3,5) but not the controls (lanes 2,4). After an additional 5 or $15 \mathrm{~min}$ with or without injected Spätzle, cytoplasm was collected and assayed for Cactus protein as described in Fig. 1. In the absence of Spätzle (lanes $2,4)$ Cactus protein accumulated, whereas in embryos exposed to Spätzle for 5 or 15 min (lanes 3,5), the newly synthesized Cactus protein was degraded. The arrow points to the Cactus protein; the high molecular weight band was used as a loading control.

bryo: signal-dependent and signal-independent degradation. Signal-independent degradation eliminates free Cactus, so that essentially all of the Cactus in the embryo is complexed with Dorsal. Signal-dependent degradation destroys. Cactus, whether or not it is complexed with Dorsal. Signal-dependent degradation of Cactus must be more rapid than signal-independent degradation, because we observed that the amount of Cactus in embryos lacking Dorsal was decreased dramatically by exposure to signal, even though both injected and control embryos were subject to signal-independent degradation (e.g., cf. Fig. 2, lanes 4 and 5).

\section{A more stable mutant form of Cactus inhibits signaling}

These experiments showed that Cactus protein stability is controlled by both signal-dependent and signal-independent degradation but did not test whether degradation of Cactus is necessary for signaling. We isolated an unusual allele of cactus, cact $^{\text {su }}$, on the basis of its ability to block signaling. This mutant allowed us to investigate the importance of Cactus stability in proper signaling.

In a large-scale genetic screen for suppressors of a dominant, ventralizing allele of easter, $e a^{831}$, one suppressor mutation was isolated. easter acts genetically upstream of Spätzle and is homologous to serine proteases (Chasan and Anderson 1989; Morisato and Anderson 1994). All of the embryos $(100 \%)$ laid by $e a^{831} /+$ females are moderately ventralized (Jin and Anderson 1990), whereas Su/ +; $e a^{831} /+$ females produced $50 \%$ wild-type, hatching lar- 
vae and $50 \%$ unhatched embryos with an apparently wild-type cuticular phenotype (Fig. 3A). The Su mutation also suppresses ventralizing mutations of several other dorsal group genes, including Toll (Fig. 3B), spätzle, and cactus (data not shown), demonstrating that the suppressor is not specific to easter but, instead, inhibits the activity of the pathway.

The Su mutation mapped by recombination to the second chromosome between $b$ and $p r$, a region including the dorsal and cactus genes. However, the Su mutation complemented both dorsal and cactus, as well as all available deficiencies in this interval, suggesting that it could be a gain-of-function allele. To obtain loss-of-function alleles at this locus we carried out a genetic screen to revert the suppressing effect of the mutation (Materials and methods). We obtained four revertants that were no longer able to suppress $e a^{831}$ and mapped to the same site as Su. Three of these revertants failed to complement loss-of-function cactus alleles. We therefore concluded that the $S u$ mutation was a gain-of-function allele of cactus and renamed it cact ${ }^{\mathrm{Su}}$.

Despite the ability of $\mathrm{cact}^{\mathrm{su}}$ to act as a strong dominant suppressor, it had no phenotype on its own: cact $^{s u} /$ cact $^{S u}$ flies are fully viable, and females produce wildtype embryos. The lack of an obvious effect on dorsalventral patterning by $\mathrm{cact}^{S u}$ was perplexing, given its strong suppression of ventralization. To look for more subtle effects of the mutation, we examined the Dorsal gradient in embryos from $\mathrm{cact}^{\mathrm{Su}} / \mathrm{cact}^{S u}$ females by examining sections stained with an anti-Dorsal antibody. Figure 4 shows that there is a reduction in the extent of the dorsal gradient in $\mathrm{cact}^{S u} / \mathrm{cact}^{S u}$ embryos compared with wild type. In wild-type embryos, $27 \%$ of the nuclei in the embryo circumference had high levels of nuclear Dorsal, whereas in $c a c t^{S u} / \mathrm{cact}^{S u}$ embryos this was reduced to $20 \%$. The absence of a later embryonic phenotype indicates that there is enough regulative capacity in the patterning process to compensate for this reduction in the number of Dorsal-containing nuclei.

\section{Cact ${ }^{\mathrm{Su}}$ encodes a slightly truncated protein that appears to be more stable than wild-type Cactus}

To determine the molecular basis of the cact ${ }^{\text {Su }}$ activity, we used reverse transcription-PCR to amplify the cactus mRNA from ovarian RNA of $\mathrm{cact}^{S u}$ females. The sequence revealed that the $\mathrm{cact}^{S u}$ allele has a single base pair change in the $5^{\prime}$ splice donor of intron six, which should render this splice site defective. The predicted protein lacks the seventh exon of the maternal cactus mRNA, which encodes 23 amino acids, and in its place has 5 amino acids encoded by intron 6 (Fig. 5A). This mutant protein is almost identical to the zygotic form of Cactus, a naturally occurring splicing variant expressed later in development (Fig. 5A; Kidd 1992).

To characterize the $\mathrm{Cact}^{\mathrm{Su}}$ protein, we performed Western blots of 0 - to 2 -hr embryo extracts from embryos laid by wild-type, $\mathrm{cact}^{S u} /+$, and $\mathrm{cact}^{\mathrm{Su}} / \mathrm{cact}^{\mathrm{Su}} \mathrm{fe}-$ males. As predicted from the mRNA sequence, the $\mathrm{Cact}^{\mathrm{Su}}$ protein is smaller than the wild-type maternal protein (Fig. 5B). Unexpectedly, we found that in embryos from $\mathrm{cact}^{\mathrm{Su}} /+$ heterozygous females, there was 10 times as much Cact ${ }^{\mathrm{su}}$ protein as wild-type protein (Fig. $5 \mathrm{~B}$, lane 2), even though the amounts of wild-type and cact $^{S u}$ RNAs in these embryos were equal /data not
A

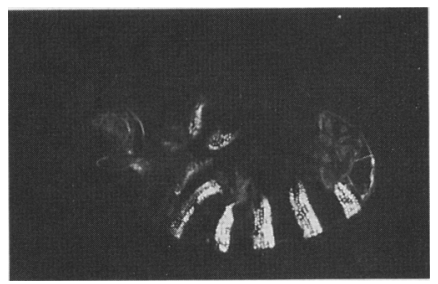

B

Figure 3. The $S u$ mutation suppresses ventralizing alleles of easter and Toll. Su was recovered in a screen for suppressors of a dominant ventralizing allele of easter, $e a^{831}$. Embryos produced by $e a^{831} /+$ females are moderately ventralized, whereas those produced by $S u /+; e a^{831} /+$ females either hatched or appeared wild type $(A)$. Su also suppressed the dominant phenotype of $T 7^{84 c}$ $(B)$, as well as ventralizing alleles of spätzle and cactus (data not shown). $\frac{e a 83 l}{+}$


$\frac{T l^{84 c}}{+}$ 


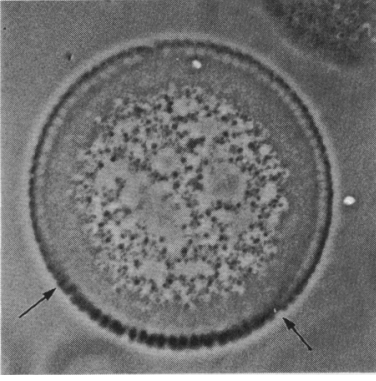

Wild Type

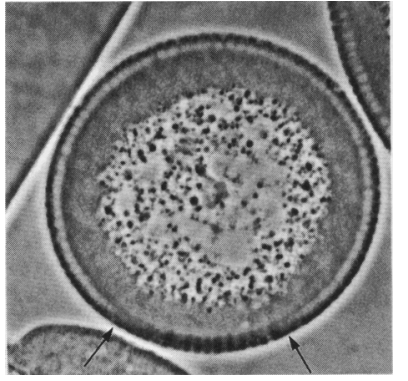

$\operatorname{cact}^{S u} /$ cact $^{S u}$
Dorsal-stained nuclei
$27 \%$
$20 \%$
Figure 4. $\mathrm{cact}^{\mathrm{Su}}$ narrows the Dorsal gradient. Embryos (2.5-3.5 hr) laid by wildtype or $\mathrm{cact}^{\mathrm{su}} / \mathrm{cact}^{\mathrm{su}}$ females were stained with Dorsal antibody and then sectioned. by arrows) was calculated as the percentage of nuclei around the embryonic circumference that stained strongly for nuclear Dorsal. In the wild-type embryos, $27 \pm 2 \%$ of the nuclei 24 of 91 in this section) contained Dorsal, whereas in embryos from $\mathrm{cact}^{S u} / \mathrm{cact}^{\text {su }}$ females only $20 \pm 2 \%$ of the nuclei (17 of 94 in this section) contained Dorsal. Four to six sections of each embryo were counted. Eight wild-type embryos and five $\mathrm{cact}^{\mathrm{St}} / \mathrm{cact}^{\mathrm{Su}}$ embryos were scored, and the results of all sections were averaged for each genotype. The extent of the Dorsal gradient (marked shown). Based on the observation that Cactus is unstareflect its greater stability. Maternal Cactus is not detected in Western blots from $\mathrm{dl}^{-}$ovarian extracts (Kidd 1992, Whalen and Steward 1993; see also Fig. 2), presumably reflecting the instability of Cactus when not comble, the greater accumulation of $\mathrm{Cact}^{\mathrm{Su}}$ protein could plexed with Dorsal. If there is normally an equilibrium between the Dorsal/Cactus complex and free subunits, then any free Cactus protein would be a target for degradation and newly synthesized Cactus could take its place in the complex. If $\mathrm{Cact}^{\mathrm{Su}}$ represents a more stable protein than wild type, it should eventually replace wild
A
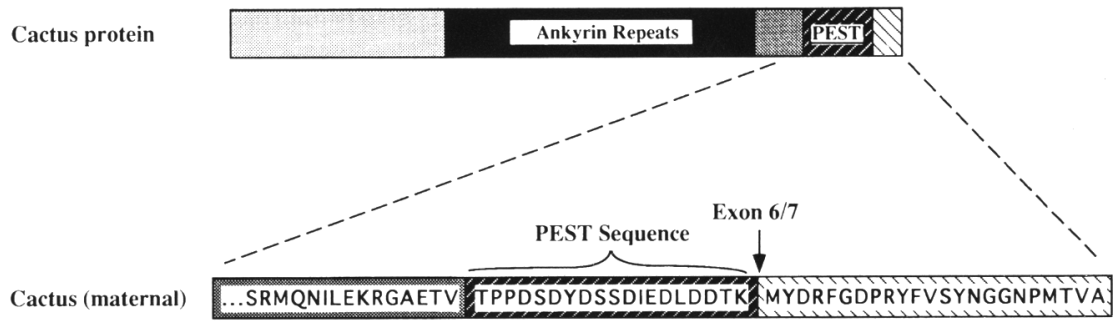

Cactus (zygotic)

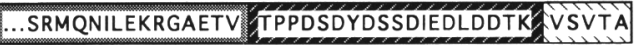

$\mathrm{Cact}^{\mathrm{Su}}$ .SRMQNILEKRGAETV TPPDSDYDSSDIEDLDDTK SESVTA
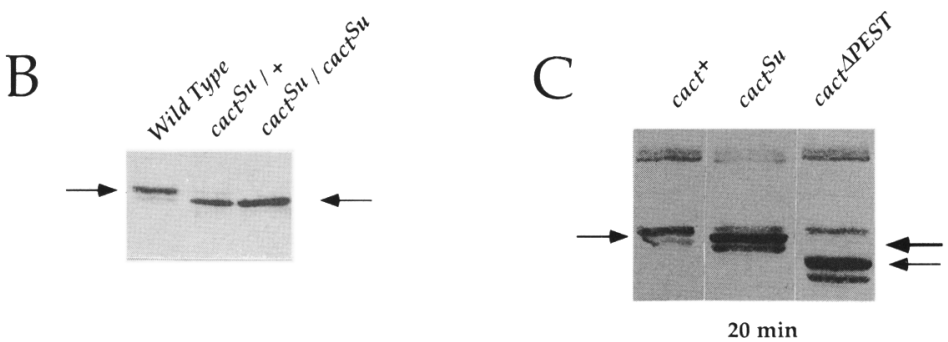
$\mathrm{cact}^{\mathrm{Su}}$ and $\mathrm{cact}^{\Delta \mathbf{P E S T}}$. $(A)$ Schematic diagram of the wild-type maternal Cactus, zygotic Cactus, $\mathrm{Cact}^{\mathrm{Su}}$, and Cact ${ }^{\mathrm{APEST}}$ proteins (drawing is not to scale). The ANKYRIN domain is a set of five conserved ankyrin repeats. The PEST domain is a region rich in Pro, Glu, Asp, Ser and Thr. The first residue after the PEST sequence in $\mathrm{Cact}^{\mathrm{Su}}$ is Glu rather than Val in zygotic Cactus because of the EMS-induced base change $(T \rightarrow A$ at the second nucleotide in intron 6) at the splice junction. Cact ${ }^{\triangle P E S T}$ lacks the entire PEST sequence, as well as the carboxy-terminal region deleted in $\mathrm{Cact}^{\mathrm{Su}}$. (B) Cactus Western blot of early embryo extracts from 0 - to 2 -hr embryos produced by wild-type, $c a c t^{\mathrm{Su}} /+$, or $c a c t^{\mathrm{Su}} /$ $\mathrm{cact}^{S u}$ females. Arrows point to the wild-type Cactus (lane 1) and Cact ${ }^{\text {su }}$ (lane 3) proteins. (Lane 2) In embryos from $\mathrm{cact}^{\mathrm{Su}} /+$ heterozygotes, there was $\sim 10$ times more $\mathrm{Cact}^{\mathrm{su}}$ protein than wild-type Cactus protein. (C) Cactus Western blot of cytoplasm from wildtype embryos injected with identical concentrations of $\mathrm{cact}^{+}, \mathrm{cact}^{\mathrm{Su}}$, and cact $^{\text {APEST }}$ RNA (Materials and methods), followed by a 20 -min incubation to allow translation of the transcripts. The left-hand arrow points to the endogenous wild-type Cactus; the right-hand arrows point to the Cact ${ }^{\text {Su }}$ proteins in lane 2 (large arrow) and the Cact ${ }^{\triangle \mathrm{PEST}}$ proteins in lane 3 . After $20 \mathrm{~min}$ of translation there is approximately three times more Cact ${ }^{\Delta \mathrm{PEST}}$ protein than wild-type Cactus present as calculated by densitometry. Note that both Cact ${ }^{\text {Su and Cact }}{ }^{\Delta \mathrm{PEST}}$ accumulate phosphorylated and unphosphorylated forms, indicating that this phosphorylation site(s) is not located in the carboxyl terminus.
Figure 5. Molecular characterization of 
type Cactus, yielding a ratio of $\mathrm{Cact}^{\mathrm{Su}} /$ wild-type Cactus similar to what we observed.

\section{Deleting the PEST sequence stabilizes Cactus and strongly inhibits signaling}

The amino acids deleted in $\mathrm{Cact}^{\mathrm{Su}}$ lie just carboxy-terminal to the PEST region of the protein (Fig. 5A; Geisler et al. 1992; Kidd 1992). PEST sequences are rich in Pro, Glu, Asp, Ser, and Thr and are thought to be associated with rapid protein turnover (Rogers et al. 1986; Rechsteiner 1990). One possible explanation for the apparently increased stability of $\mathrm{Cact}^{\mathrm{Su}}$ is that the lack of sequences immediately adjacent to the PEST region interferes with the destabilizing function of the PEST sequence. In this view, removing the PEST sequence entirely might result in an even more stable protein.

To test whether the PEST sequence does affect Cactus stability, we constructed a mutant form of cactus, $c a c t^{\Delta \mathrm{PEST}}$, in which both the seventh exon and the entire PEST sequence were deleted. The ankyrin repeat domain remained intact in this mutant (Fig. 5A). To compare the stability of the different Cactus proteins, we injected in vitro-synthesized trancripts of $\mathrm{Cact}^{+}, \mathrm{Cact}^{\mathrm{Su}}$, and $c a c t^{\Delta \mathrm{PEST}}$ into wild-type embryos and allowed them to be translated in vivo. We then collected the cytoplasm from these embryos and analyzed it by Western blot. After 20 min of translation, there was more Cact ${ }^{\triangle P E S T}$ and $\mathrm{Cact}^{\mathrm{Su}}$ than wild-type Cactus (Fig. 5C), even though equivalent concentrations of RNA were injected (Materials and methods). This suggested that Cact ${ }^{\triangle \mathrm{PEST}}$ and $\mathrm{Cact}^{\mathrm{Su}}$ are both more stable than wild- type Cactus.

To test whether cact ${ }^{\Delta \text { PEST }}$ could block signaling like cact $^{S u}$, we injected cact ${ }^{\Delta \text { PEST }}$ RNA transcripts into wildtype embryos. This resulted in weakly dorsalized embryos (Fig. 6), suggesting that the Cact ${ }^{\Delta \mathrm{PEST}}$ protein interfered with signaling. A stronger effect was seen when $c a c t^{\Delta \mathrm{PEST}}$ RNA was injected into $\mathrm{cact}^{\mathrm{Su}} / \mathrm{cact}^{\mathrm{Su}}$ embryos, where $15 \%$ of the embryos were completely dorsalized (Fig. 6). This strong dorsalization was never observed with injection of $\mathrm{Cact}^{+}$or $\mathrm{cact}^{\mathrm{Su}}$ transcripts (data not shown). Therefore, Cact ${ }^{\triangle \mathrm{PEST}}$ is more effective at blocking signaling than $\mathrm{Cact}^{\mathrm{Su}}$ and is capable of completely blocking all signaling in embryos.

\section{Cact $^{\text {Su }}$ and Cact ${ }^{\triangle P E S T}$ also degrade rapidly in response to signal}

The above experiments showed that the more stable forms of Cactus, Cact ${ }^{\text {su }}$ and Cact ${ }^{\Delta \mathrm{PEST}}$, can block signaling. In principle, the ability to block signaling could reflect resistance to either signal-dependent or signal-independent degradation of Cactus. To determine whether $\mathrm{Cact}^{\mathrm{Su}}$ and Cact ${ }^{\triangle \mathrm{PEST}}$ were more resistant to signal-dependent degradation than wild-type Cactus, we injected activated Spätzle into embryos laid by females carrying these mutants. Activated Spätzle elicited the rapid degradation of the $\mathrm{Cact}^{\mathrm{Su}}$ protein at a similar rate as the wild-type protein (cf. Figs. 7A and 1A). There was more Cact $^{\text {Su }}$ than wild-type Cactus per embryo at all time


Figure 6. cact $^{\Delta \text { PEST }}$ can block signaling. In vitro-synthesized cact $^{\Delta \text { PEST }}$ RNA was injected into embryos from wild-type or $\mathrm{cact}^{S u} / \mathrm{cact}^{\mathrm{Su}}$ females. When injected into wild-type embryos, cact $^{\Delta \text { PEST }}$ RNA causes weak dorsalization (top). When cact $^{\Delta \text { PEST }}$ was injected into embryos produced by $\mathrm{cact}^{S u} / \mathrm{cact}^{\mathrm{Su}}$ females, it caused complete dorsalization in $15 \%$ of the embryos (bottom). This strongly dorsalized embryo lacks all ventral and lateral structures and is composed of a hollow tube of dorsal cuticle. Weaker dorsalization was observed in the other $85 \%$ of injected embryos.

points, but the rate of degradation was approximately the same. Therefore, the Cact ${ }^{\mathrm{Su}}$ protein, while encoding a more stable form of Cactus, is not resistant to signaldependent degradation.

To test the response of Cact ${ }^{\triangle \mathrm{PEST}}$ to signaling, we made stable transformants of cact ${ }^{\text {PPEST }}$ (Materials and methods). When activated Spätzle was injected into embryos from transformant females carrying one copy of the cact $^{\Delta \text { PEST }}$ transgene and two wild-type copies of cactus, both $\mathrm{Cact}^{\triangle \mathrm{PEST}}$ and wild-type Cactus were degraded rapidly (Fig. 7B). Therefore, both $\mathrm{Cact}^{\mathrm{su}}$ and Cact ${ }^{\Delta \mathrm{PEST}}$ are degraded rapidly in response to signal.

\section{Cact $^{\text {Su }}$ and Cact $^{\triangle P E S T}$ are resistant to signal- independent degradation}

The previous experiments showed that $\mathrm{Cact}^{\mathrm{Su}}$ and Cact ${ }^{\Delta \text { PEST }}$ undergo normal rates of signal-dependent degradation. To test whether their greater stability might be caused by greater resistance to signal-independent degradation of free Cactus, we compared the level of protein present after injection of $\mathrm{cact}^{+}, \mathrm{cact}^{\mathrm{Su}}$, and cact ${ }^{\mathrm{APEST}}$ RNAs into embryos from $\mathrm{dl}^{-}$females prior to the normal time of signaling (Fig. 8). Because the mutant proteins were synthesized in the absence of Dorsal, and because no activated Spätzle was injected, the proteins were subject to signal-independent but not signal-dependent degradation. Thirty minutes after injection, there 

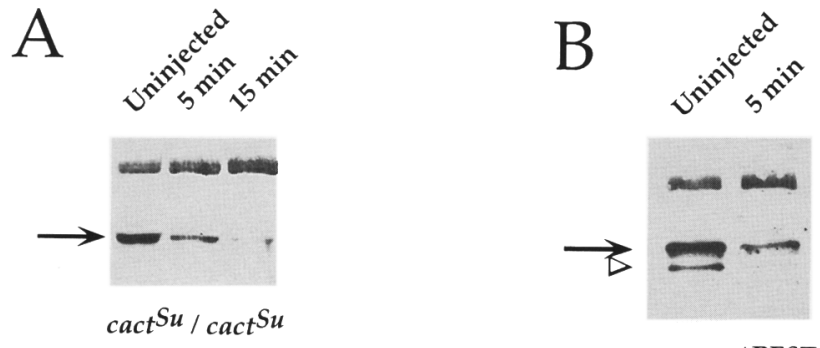

$\mathrm{P}\left[w^{+}, \operatorname{cact}^{\triangle P E S T}\right]$

Figure 7. $\mathrm{Cact}^{\mathrm{Su}}$ and Cact ${ }^{\Delta \mathrm{PEST}}$ are degraded in response to signaling. Activated Spätzle was injected into embryos produced by $\mathrm{cact}^{S u} / \mathrm{cact}^{S u}$ females $(A)$ or $\mathrm{P}\left[W^{+}{ }^{\text {, }}\right.$ cact $\left.^{\Delta \text { PEST}}\right]$ females $(B)$. The arrow in $A$ points to the Cact ${ }^{\mathrm{Su}}$ protein. Cact $^{\mathrm{Su}}$ degrades rapidly in response to signaling: $15 \mathrm{~min}$ following injection there is still some detectable Cact ${ }^{\text {Su }}$ protein visible (lane 3), but its level is greatly reduced compared with uninjected embryos (lane 1). In $B$, embryos laid by transformant females carrying one copy of cact ${ }^{\text {APEST }}$ and two copies of wild-type cactus were injected with activated Spätzle. This transformant line produces substantially less Cact ${ }^{\Delta \mathrm{PEST}}$ protein than wild-type Cactus. The arrow points to the wild-type Cactus protein; the open arrowhead points to the Cact ${ }^{\triangle \mathrm{PEST}}$ protein. Lane 1 contains cytoplasm from uninjected embryos. Five minutes after injection of activated Spätzle (lane 2), the levels of both wild-type Cactus and Cact $^{\triangle \mathrm{PEST}}$ are greatly reduced.

was much more Cact ${ }^{\mathrm{Su}}$ and $\mathrm{Cact}^{\mathrm{APEST}}$ protein present than wild-type Cactus (Fig. 8, lanes 3,6,9) even though equivalent amounts of RNA were injected (Materials and methods). Because cact $^{S u}$ contains the entire wild-type 5 '- and 3 '-untranslated regions, the observed difference in protein levels is most likely the result of the greater stability of the Cact ${ }^{\mathrm{Su}}$ and $\mathrm{Cact}^{\mathrm{\Delta PEST}}$ proteins rather than differential translation. This result suggests that the Cact ${ }^{\mathrm{Su}}$ and Cact ${ }^{\triangle \mathrm{PEST}}$ proteins are relatively insensitive to signal-independent degradation and that it is the resultant increase in free Cactus in the embryo that inhibits signaling.

\section{Discussion}

\section{Signal-dependent degradation of Cactus}

We have shown that Cactus is rapidly degraded in response to stimulation of the Drosophila dorsal-ventral signaling pathway. The mammalian homolog of Cactus, I $\mathrm{B}$, is degraded in response to signaling in tissue culture cells (Beg et al. 1993; Henkel et al. 1993; Palombella et al. 1994; Traenckner et al. 1994). Our experiments in whole embryos demonstrate that rapid signal-dependent degradation of Cactus takes place in the intact organism.

Two plausible models could explain how Cactus is degraded in response to the signal. One model is that signaling causes dissociation of the Dorsal/Cactus complex by modifying either Dorsal or Cactus, and the resulting free Cactus is degraded by signal-independent degradation (see below). The other model is that Cactus is degraded directly in the complex. Because Cactus is degraded in response to signal even in the absence of
Dorsal, we conclude that Cactus degradation is a direct target of signaling. We infer that in the wild-type embryo, Cactus in the complex is degraded and that this degradation releases Dorsal, allowing it to move to the nucleus.

All of the genetically defined components that act upstream of cactus in the pathway, Toll, tube, and pelle, are required for signal-dependent degradation of Cactus, confirming that Cactus degradation is an integral step in this signaling pathway. However, the nature of these components does not clarify how Cactus protein is degraded in response to the signal. Genetic analysis has shown that tube acts downstream of Toll (Hecht and Anderson 1993) and pelle acts downstream of tube (Grosshans et al. 1994). The pelle gene appears to encode a serine-threonine kinase (Shelton and Wasserman 1993), and because Cactus is a phosphoprotein, it could be a substrate for Pelle.

Rapid phosphorylation of IKB in response to signaling and prior to its degradation has been observed in response to IL-1 and TNF- $\alpha$ (Beg et al. 1993; Miyamoto et al. 1994; Traenckner et al. 1994). Although we found that Pelle activity is required in vivo for Cactus degradation, we did not observe phosphorylation of Cactus in response to signaling. It is possible that such a phosphorylated form exists but was not detected by our methods because of its short half-life or because it did not cause a detectable mobility shift. Alternatively, Pelle might control Cactus degradation indirectly, phosphorylating an unknown intermediate in the pathway.

Our findings that the Cact ${ }^{\text {Su }}$ and Cact ${ }^{\mathrm{SPEST}}$ mutants degrade rapidly in response to signaling demonstrate that the PEST sequence is not required for signal-dependent degradation of Cactus. This implies that there is another region of Cactus, located either in the amino terminus or

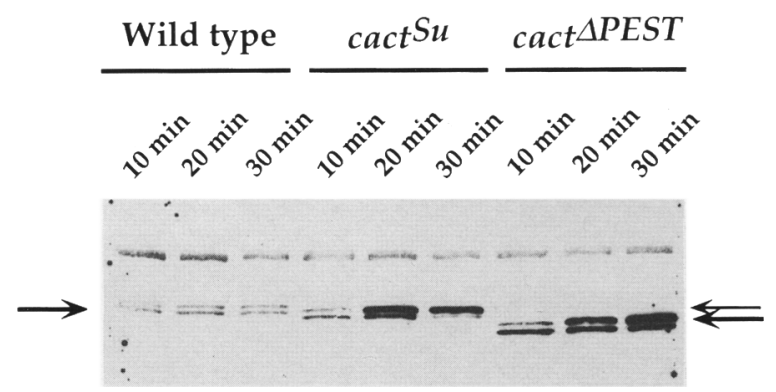

Figure 8. $\mathrm{Cact}^{\mathrm{Su}}$ and $\mathrm{Cact}^{\triangle \mathrm{PEST}}$ are relatively resistant to signal-independent degradation of free Cactus in the absence of Dorsal. In vitro-synthesized transcripts of wild type cactus, $\mathrm{cact}^{\text {su}}$, and $\mathrm{cact}^{\Delta \mathbf{P E S T}}$ were injected into embryos from $\mathrm{dl}^{-} \mathrm{fe}$ males. Cytoplasm was collected 10,20, and $30 \mathrm{~min}$ after injection and analyzed by a Cactus Western blot. Identical concentrations of the three RNAs were injected (Materials and methods). The level of Cact ${ }^{\triangle \mathrm{PEST}}$ protein that accumulates after 30 min is greater than that of $\mathrm{Cact}^{\mathrm{Su}}$ and much greater than that of wild-type Cactus (cf. lanes 3, 6, and 9). The arrows point to the Cactus proteins; the more slowly migrating band is a crossreacting band. 
in the ankyrin repeats, that is required for rapid degradation in response to signaling.

\section{Two kinds of degradation control Cactus protein levels}

We have shown that Cactus protein stability is regulated at two levels (Fig. 9). The first level of regulation is the signal-dependent degradation described above. The second is signal-independent degradation of the Cactus protein that is not complexed with Dorsal. We found that in the absence of Dorsal, the Cact ${ }^{\mathrm{Su}}$ and Cact ${ }^{\mathrm{APEST}}$ proteins accumulate to a much higher level than wild-type Cactus protein after injection of equivalent amounts of RNA. Strong evidence that the greater accumulation of $\mathrm{Cact}^{\mathrm{Su}}$ and $\mathrm{Cact}^{\triangle \mathrm{PEST}}$ reflects increased stability rather than increased translation comes from the comparison of Cactus protein levels in embryos from $\mathrm{Cact}^{\mathrm{Su}} / \mathrm{cact}^{+}$heterozygous females. In these embryos, the level of Cact ${ }^{\mathrm{su}}$ is greater than the level of $\mathrm{Cact}^{+}$, and furthermore, the level of $\mathrm{Cact}^{+}$is 10 -fold lower than in wild-type embryos. It is very unlikely that the decrease in Cact ${ }^{+}$is attributable to increased translation of $\mathrm{Cact}^{\mathrm{Su}}$. If, however, there is an equilibrium between free Cactus and Cactus in the Dorsal/Cactus complex, then because free $\mathrm{Cact}^{\mathrm{Su}}$ is more stable than free Cact ${ }^{+}$, it will accumulate preferentially over time.

Our experiments indicate that in the absence of Dorsal, wild-type Cactus turns over much more rapidly than $\mathrm{Cact}^{\mathrm{Su}}$ and $\mathrm{Cact}^{\triangle \mathrm{PEST}}$. Because these mutants lack sequences in and near the PEST region, we infer that the PEST sequence is important for the degradation of free Cactus. The mr:chanism by which PEST sequences cause protein degradation is unknown; however, our finding that $\mathrm{Cact}^{\mathrm{Su}}$, which retains the entire PEST sequence but lacks the adjacent amino acids, is more stable than wildtype Cactus suggests that the conformation of the PEST region is important for its function.

\section{Increasing the amount of free Cactus blocks signaling}

The normal consequence of signal-independent degradation is to remove nearly all Cactus protein that is not complexed with Dorsal from the embryo. The Cact ${ }^{\text {su }}$ and Cact ${ }^{\triangle \mathrm{PEST}}$ proteins, which are resistant to signalindependent degradation, can effectively block signaling, revealing that excess free Cactus interferes with normal signaling. Free Cactus could block signaling in one of two ways. It could act as a buffer, binding to Dorsal after it is freed from the complex, decreasing its probability of translocating to the nucleus. Alternatively, free Cactus could compete with the Cactus in the Dorsal/Cactus complex for a limiting upstream component that targets Cactus for degradation, effectively titrating out the signal from upstream in the pathway.

A standing controversy in the field is whether the dorsal-ventral signaling pathway acts by modifying Dorsal, Cactus, or both. Our results show that signal-dependent degradation of Cactus does not require Dorsal, indicating that Cactus is a direct target of the pathway and suggesting that all signaling could be mediated via Cactus. However, even the strongest cactus mutants retain some residual dorsal-ventral polarity, with more Dorsal protein translocated into ventral than dorsal embryonic nuclei (Roth et al. 1991). Because we obtained completely dor-
Figure 9. Model of the two forms of regulation of Cactus protein degradation. (Amino) Aminoterminus of Cactus; (ANK) ankyrin repeat domain; (P) PEST region. Signal-dependent degradation of Cactus, which requires the action of Toll, tube, and pelle, causes degradation of Cactus in the Dorsal/Cactus complex, leading to the liberation of free Dorsal, which then migrates into ventral nuclei. This type of degradation is mediated by more amino-terminal regions, because the Cact ${ }^{\Delta \text { PEST }}$ mutant is subject to signal-dependent degradation. (Right) Free Cactus that is not complexed with Dorsal is degraded by signal-independent degradation. The PEST sequence is important for this process, as the Cact $^{\triangle P E S T}$ mutant that lacks a PEST sequence is resistant to signal-independent degradation.




salized embryos with the stabilized forms of Cactus protein, this demonstrates that excess Cactus can effectively block all signaling. We therefore conclude that degradation of Cactus is necessary for any nuclear translocation of Dorsal. Once Cactus is degraded, the additional modification of free Dorsal that has been observed (Gillespie and Wasserman 1994) may be required for Dorsal to achieve the highest nuclear concentrations.

The observation that Cactus, like IKB, is rapidly degraded in response to signaling strengthens the parallels between the mammalian and Drosophila signaling pathways. Given the similarities that exist between the two systems, our findings on Cactus may also apply to IкB. It should now be possible to test whether the level of IкB protein, like Cactus, is controlled by both signal-dependent and signal-independent processes, whether normal signaling targets IкB directly for degradation, and whether there is a specific kinase required for $\mathrm{I}_{\kappa} \mathrm{B}$ degradation. We plan to continue to exploit the genetic interactions in Drosophila to elucidate the mechanisms of signal-dependent protein degradation.

\section{Materials and methods}

\section{Fly strains and mutagenesis}

Oregon- $\mathrm{R}$ was used as the wild-type stock. The easter alleles are described in Chasan and Anderson (1989) and Jin and Anderson (1990). For $T 1^{-}, t u b^{-}, \mathrm{pll}^{-}$, and $\mathrm{dl}^{-}$females: $T 1^{5 B R E Q}$, $T l^{9 Q R E}$ (Anderson et al. 1985), tub ${ }^{R 5.6} / \mathrm{Df}(3 R) X M 3, \mathrm{pll}^{74}$ / $D f(3 R)$ Ser $^{+R 82 f}$ (Hecht and Anderson 1993), and $d l^{8} / D f(2 L) 119$ (Roth et al. 1989) were used. Ethylmethane sulfonate (EMS) mutagenesis was carried out as described (Lewis and Bacher 1968). To isolate suppressors of $e a^{831}$, mutagenized $e a^{831}$, knirps/TM3, tld ${ }^{10 E}$ males were crossed to $e a^{1}, t l d^{10 E} / \mathrm{TM} 1$, knirps females. Balancers carrying the zygotic lethal mutations had been isolated previously (Ferguson and Anderson 1992). The only viable progeny from this cross were $e a^{1}, t 1 d^{10 E} / e a^{831}$, knirps males and females, which were placed in bottles and checked for fertility. The presence of $e a^{1}$ in these flies ensured that intragenic suppressors would not be recovered, as $e a^{1} / e a^{-}$females are sterile. Of $75,000 \mathrm{~F}_{1}$ females, one was fertile, which proved to be $\mathrm{cact}^{\mathrm{Su}}$. In the screen to generate revertants of $\mathrm{cact}^{\mathrm{Su}}$, a transformant line was used that contained a dominant allele of easter, e $a^{831-}$ 125.3 inserted on the second chromosome (Jin 1991). cact $^{\mathrm{Su}} / \mathrm{CyO}$ males were mutagenized with EMS and crossed to + /CyO females. Four thousand single $\mathrm{F}_{1}$ females of the genotype $\mathrm{cact}^{S u^{*}} /$ CyO were crossed to $e a^{831-125.3} / \mathrm{CyO}$ males. The embryos laid by $\mathrm{cact}^{\mathrm{Su}} / e a^{831-125.3} \mathrm{~F} 2$ females were scored for ventralization. Four revertants were recovered; all four mapped to $\mathrm{cact}^{\mathrm{Su}}$, and three of these were ventralized over $\operatorname{cact}^{99}$ (Roth et al. 1991).

\section{Cuticle preparations}

Embryos were collected for $24 \mathrm{hr}$ and aged for another $24 \mathrm{hr}$ to allow completion of embryonic development. The embryos were then dechorionated in bleach, dissected out of their vitelline membranes, fixed in 1:4 glycerol/acetic acid, and mounted in Hoyer's medium diluted 1:1 with lactic acid. Injected embryos were allowed to develop for $36 \mathrm{hr}$ after injection before cuticle preparations were made.

\section{Embryo injections}

Synthetic SP6 RNA transcripts were generated by in vitro tran- scription carried out in the presence of radioactively labeled ATP as described (Schneider et al. 1991) with the modification that two sequential $5 \mathrm{M}$ ammonium acetate/isopropanol precipitations were carried out followed by Cerenkov counting. The transcripts were diluted as necessary to ensure equivalent concentrations. For RNA injections, transcripts were injected into 1- to 1.5-hr embryos (Chasan and Anderson 1989). Embryos were allowed to develop for cuticle preparations, or the cytoplasm was collected for Western analysis. Cytoplasm was collected by aspirating the contents of 15 embryos into the injection needle, avoiding any cytoplasm that had leaked out of the embryo after injection. The cytoplasm was deposited into the injection oil where it was recovered with a pipette and placed immediately in buffer on ice. The buffer was a 1:1 dilution of 20 mM HEPES $(\mathrm{pH} 7.5), 20 \mathrm{mM} \mathrm{NaCl}, 1 \mathrm{mg} / \mathrm{ml}$ of aprotinin, 1 $\mathrm{mg} / \mathrm{ml}$ of leupeptin, $1 \mathrm{mg} / \mathrm{ml}$ of antipain, in $2 \times$ protein sample buffer (Ausubel et al. 1991) containing 10\% 2-mercaptoethanol. A crude activated Spätzle preparation was made (Schneider et al. 1994), and a concentration equivalent to $150 \mathrm{U} / \mathrm{ml}$, as described in Schneider et al. (1994) was used. The activated Spätzle injections were carried out in 2- to 2.5 -hr embryos. For the sequential RNA and activated Spätzle injections, RNA was first injected into the cytoplasm as described above. The activated Spätzle was then injected into the perivitelline space on the dorsal side of the embryo as described (Schneider et al. 1994) using the same point of entry as for the RNA. Cytoplasm was collected for Western analysis as described above.

\section{Antibody staining of embryos}

Embryos (2.5- to 3.5-hr) were prepared and stained as described (Roth et al. 1989) using a 1:1000 dilution of rat anti-Dorsal antibody received from $R$. Steward (Rutgers University, Piscataway, NI). A goat anti-rat-horseradish peroxidase (HRP) secondary antibody was used; staining was detected with DAB. Embryos were embedded in Durcupan (Fluka) resin and sectioned $(10 \mu \mathrm{m})$.

\section{Cactus constructs and transformants}

To sequence $\mathrm{cact}^{\mathrm{Su}}$, ovarian RNA was isolated (Ashburner et al. 1989a) from $\mathrm{cact}^{\mathrm{Su}} / \mathrm{cact}^{\mathrm{Su}}$ females. An oligonucleotide (GAT CGA ATT CTT CTG CAT CCT TGT ATG CTT TA/ directed against the $3^{\prime}$ end of the cactus cDNA was used as a primer for reverse transcriptase, and RT-PCR was carried out (Ausubel et al. 1991) using this primer as the $3^{\prime}$ primer and GAT CGG ATC CAT TCG CTA TCG AAA CGT G as the $5^{\prime}$ primer. The $5^{\prime}$ primer contained a BamHI site, and the $3^{\prime}$ primer contained an EcoRI site for cloning into pSP64Poly(A) (Promega). Sequencing was performed according to Ausubel et al. (1991.) $\mathrm{Cact}^{\mathrm{APEST}}$ was constructed by PCR mutagenesis using TTT GCC GTG CCA AAC GAA as the $5^{\prime}$ primer and ACT GTC CGG ATC ATC ATA CGG TCTC as the $3^{\prime}$ primer. This inserted two tandem stop codons just prior to the PEST sequence. AgeI, a site just downstream of the stop codons, and BspEI, an upstream site, were used to clone the mutant fragment into the wild-type vector. CaSpeR and $\mathrm{p} \pi 25.7 \mathrm{wc}$ were used to generate stable transformants (Ashburner 1989b).

\section{Western blots}

Samples were boiled for $5 \mathrm{~min}$, spun at 13,000 rpm for $2 \mathrm{~min}$, and run on a $7 \%$ polyacrylamide gel. The proteins were transferred onto nitrocellulose (Sigma) and probed with 1:1000 mouse anti-Cactus antibody from S. Kidd as described (Ausubel et al. 1991). A goat anti-mouse HRP-conjugated antibody (Bio- 
Rad) was used as the secondary antibody, and ECL (Amersham) was used for detection. Scanning densitometry was used to measure band densities.

\section{Acknowledgments}

We are very grateful to Simon Kidd for providing Cactus antibody. We thank Ruth Steward for providing Dorsal antibody and Robert Geisler for sending the sequence of Cactus prior to its publication. We thank Tanya Wolff, Don Rio, and members of the Anderson laboratory, especially Sima Misra, for helpful comments on the manuscript. This work was supported by an American Cancer Society grant (DB-42D) to K.V.A. and a National Institutes of Heath predoctoral training grant to M.P.B.

The publication costs of this article were defrayed in part by payment of page charges. This article must therefore be hereby marked "advertisement" in accordance with 18 USC section 1734 solely to indicate this fact.

\section{References}

Anderson, K.V., G. Jürgens, and C. Nüsslein-Volhard. 1985. Establishment of dorsal-ventral polarity in the Drosophila embryo: Genetic studies on the role of the Toll gene product. Cell 42: 779-789.

Ashburner, M. 1989a. Drosophila: A laboratory manual. Cold Spring Harbor Laboratory Press, Cold Spring Harbor, New York.

- 1989b. Drosophila: A laboratory handbook. Cold Spring Harbor Laboratory Press, Cold Spring Harbor, New York.

Ausubel, F.M., R. Brent, R.E. Kingston, D.D. Moore, J.G. Seidman, J.A. Smith, and K. Struhl. 1991. Current protocols in molecular biology. John Wiley and Sons/Greene, New York.

Baeuerle, P.A. and D. Baltimore. 1988. Activation of DNA-binding activity in an apparently cytoplasmic precursor of the NF-kB transcription factor. Cell 53: 211-217.

Beg, A.A. and A.S. Baldwin Jr. 1993. The IkB proteins: Multifunctional regulators of $\mathrm{Rel} / \mathrm{NF}-\mathrm{kB}$ transcription factors. Genes \& Dev. 7: 2064-2070.

Beg, A.A., T.S. Finco, P.V. Nantermet, and A.S. Baldwin Jr. 1993. Tumor necrosis factor and interleukin-1 lead to phosphory-

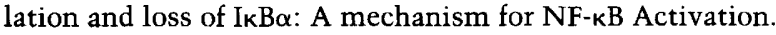
Mol. Cell Biol. 13: 3301-3310.

Blank, V., P. Kourilsky, and A. Israël. 1992. NF-кB and related proteins: Rel/dorsal homologies meet ankyrin-like repeats. Trends Biochem. Sci. 17: 135-140.

Chasan, R. and K.V. Anderson. 1989. The role of easter, an apparent serine protease, in organizing the dorsal-ventral pattern of the Drosophila embryo. Cell 56: 391-400.

Ferguson, E.L. and K.V. Anderson. 1992. Localized enhancement and repression of the activity of the TGF- $\beta$ family member, decapentaplegic, is necessary for dorsal- ventral pattern formation in the Drosophila embryo. Development 114: $583-597$.

Ganchi, P.A., S.C. Sun, W.C. Greene, and D.W. Ballard. 1992. I $\mathrm{B} / \mathrm{MAD}-3$ masks the nuclear localization signal of NF- $\mathrm{B}$ p65 and requires the transactivation domain to inhibit NFкB p65 DNA binding. Mol. Biol. Cell 3: 1339-1352.

Geisler, R., A. Bergmann, Y. Hiromi, and C. Nüsslein-Volhard. 1992. cactus, a gene involved in dorsoventral pattern formation of Drosophila, is related to the I $\mathrm{KB}$ gene family of vertebrates. Cell 71: 613-621.

Gillespie, S.K. and S.A. Wasserman. 1994. dorsal, a Drosophila Rel-like protein, is phosphorylated upon activation of the transmembrane protein Toll. Mol. Cell. Biol. 14: 3559-3568.
Grilli, M., J.J.-S. Chiu, and M.J. Lenardo. 1993. NF-кB and Rel: Participants in a multiform transcriptional regulatory system. Int. Rev. Cytol. 143: 1-62.

Grosshans, J, A. Bergmann, P. Haffter, and C. Nüsslein-Volhard. 1994. Activation of the kinase Pelle by Tube in the dorsoventral signal transduction pathway of Drosophila embryo. Nature 372: 563-566.

Hashimoto, C., K.L. Hudson, and K.V. Anderson. 1988. The Toll gene of Drosophila, required for dorsal-ventral embryonic polarity, appears to enode a transmembrane protein. Cell 52: 269-279.

Hecht, P.M. and K.V. Anderson. 1993. Genetic characterization of tube and pelle, genes required for signaling between Toll and dorsal in the specification of the dorsal-ventral pattern of the Drosophila embryo. Genetics 135: 405-417.

Henkel, T., U. Zabel, K. van Zee, J.M. Müller, E. Fanning, and P.A. Baeuerle. 1992. Intramolecular masking of the nuclear location signal and dimerization domain in the precursor for the p50 NF-кB subunit. Cell 68: 1121-1133.

Henkel, T., T. Machleidt, I. Alkalay, M. Krönke, Y. Ben-Neriah, and P.A. Baeuerle. 1993. Rapid proteolysis of IкB- $\alpha$ is necessary for activation of transcription factor NF- $\mathrm{kB}$. Nature 365: $182-185$.

Jin, Y. 1991. "Generating dorsal-ventral asymmetry in the Drosophila embryo: Studies on the easter gene and the polarizing activity." Ph.D. thesis, University of Calfornia at Berkeley, Berkeley, CA.

Jin, Y. and K.V. Anderson. 1990. Dominant and recessive alleles of the Drosophila easter gene are point mutations at conserved sites in the serine protease catalytic domain. Cell 60: $873-881$.

Kidd, S. 1992. Characterization of the Drosophila cactus locus and analysis of interactions between cactus and dorsal Proteins. Cell 71: 623-635.

Letsou, A., S. Alexander, K. Orth, and S.A. Wasserman. 1991 Genetic and molecular characterization of tube, a Drosophila gene maternally required for embryonic dorsoventral polarity. Proc. Natl. Acad. Sci. 88: 810-814.

Lewis, E.B. and F. Bacher. 1968. Methods of feeding ethyl methane-sulfonate (EMS) to Drosophila males. Dros. Inf. Serv. 43: 193.

Lin, Y.-C., K. Brown, and U. Siebenlist. 1995. Activation of NF$\kappa \mathrm{B}$ requires proteolysis of the inhibitor IкB- $\alpha$ : Signal-induced phosphorylation of IкB- $\alpha$ alone does not release active NFкB. Proc. Natl. Acad. Sci. 92: 552-556.

Miyamoto, S., M. Maki, M.J. Schmitt, M. Hatanaka, and I.M. Verma. 1994. Tumor necrosis factor $\alpha$-induced phosphorylation of $\mathrm{I} \mathrm{K} \mathrm{\alpha} \alpha$ is a signal for its degradation but not dissociation from NF-кB. Proc. Nat1. Acad. Sci. 91: 12740-12744.

Morisato, D. and K.V. Anderson. 1994. The spätzle gene encodes a component of the extracellular signaling pathway establishing the dorsal-ventral pattern of the Drosophila embryo. Cell 76: 677-688.

Palombella, V.J., O.J. Rando, A.L. Goldberg, and T. Maniatis. 1994. The ubiquitin- proteasome pathway is required for processing the NF- $\mathrm{kB} 1$ precursor protein and the activation of NF-кB. Cell 78: 773-785.

Rechsteiner, M. 1990. PEST sequences are signals for rapid intracellular proteolysis. Semin. Cell Biol. 6: 433-440.

Rogers, S., R. Wells, and M Rechsteiner. 1986. Amino acid sequences common to rapidy degraded proteins: The PEST hypothesis. Science 234: 364-368.

Roth, S., D. Stein, and C. Nüsslein-Volhard. 1989. A gradient of nuclear localization of the dorsal protein determines dorsoventral pattern in the Drosophila embryo. Cell 59: 11891202. 
Roth, S., Y. Hiromi, D. Godt, and C. Nüsslein-Volhard. 1991. cactus, a maternal gene required for proper formation of the dorsoventral morphogen gradient in Drosophila embryos. Development 112: 371-388.

Schneider, D.S., K.L. Hudson, T.Y. Lin, and K.V. Anderson. 1991. Dominant and recessive mutations define functional domains of Toll, a transmembrane protein required for dorsal-ventral polarity in the Drosophila embryo. Genes $\Theta$ Dev. 5: 797-807.

Schneider, D.S., Y. Jin, D. Morisato, and K.V. Anderson. 1994. A processed form of the Spätzle protein defines dorsal-ventral polarity in the Drosophila embryo. Development 120: 12431250.

Sen, R. and D. Baltimore. 1986. Inducibility of $\kappa$ immunoglobulin enhancer-binding protein NF- $\mathrm{B}$ by a posttranslational mechanism. Cell 47: 921-928.

Shelton, C.A. and S.A. Wasserman. 1993. pelle encodes a protein kinase required to establish dorsoventral polarity in the Drosophila embryo. Cell 72: 515-525.

Steward, R. 1987. Dorsal, an embryonic polarity gene in Drosophila, is homologous to the vertebrate proto-oncogene, c-rel. Science 238: 692-694.

Traenckner, E.B.-M., S. Wilk, and P.A. Baeuerle. 1994. A proteasome inhibitor prevents activation of NF- $\mathrm{kB}$ and stabilizes a newly phosphorylated form of IKB- $\alpha$ that is still bound to NF-кB. EMBO $/$. 13: 5433-5441.

Whalen, A.M. and R. Steward. 1993. Dissociation of the DorsalCactus complex and phosphorylation of the Dorsal protein correlate with the nuclear localization of Dorsal. J. Cell Biol. 123: $523-534$. 


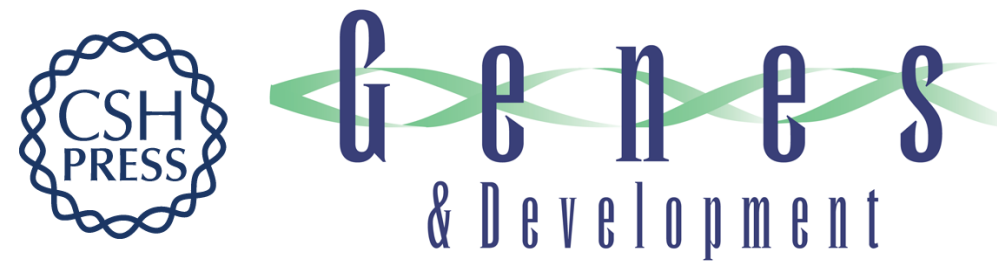

\section{Cactus protein degradation mediates Drosophila dorsal-ventral signaling.}

M P Belvin, Y Jin and $\mathrm{K} V$ Anderson

Genes Dev. 1995, 9:

Access the most recent version at doi:10.1101/gad.9.7.783

References This article cites 35 articles, 15 of which can be accessed free at:

http://genesdev.cshlp.org/content/9/7/783.full.html\#ref-list-1

License

Email Alerting

Service

Receive free email alerts when new articles cite this article - sign up in the box at the top right corner of the article or click here.

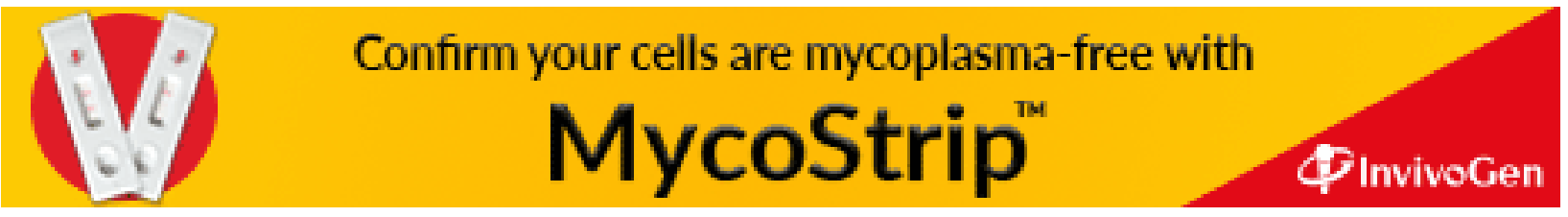

